E11-2008-160

I. G. Christov ${ }^{1}$, S. N. Dimova ${ }^{1}$, T. L. Boyadjiev ${ }^{2}$

\title{
STABILITY AND BIFURCATION OF THE MAGNETIC FLUX BOUND STATES IN STACKED JOSEPHSON JUNCTIONS
}

Presented at the Fourth International Conference on Numerical Analysis and Applications, NAA-2008, Lozenetz, Bulgaria

\footnotetext{
${ }^{1}$ University of Sofia, Bulgaria

${ }^{2}$ University of Sofia, Bulgaria \& JINR, Dubna, Russia
} 
Христов И.Г., Димова С. Н., Бояджиев Т.Л.

Устойчивость и бифуркации статических распределений

магнитного потока в многослойных джозефсоновских контактах

Рассмотрен эффективный алгоритм для решения нелинейных краевых задач для систем ОДУ, моделирующих распределения магнитного потока в многослойных джозефсоновских контактах. Алгоритм основывается на непрерывном аналоге метода Ньютона. Возникающие на каждой итерации линейные краевые задачи решаются численно методом конечных элементов. Глобальная устойчивость распределений исследуется при помощи матричной задачи Штурма-Лиувилля. В качестве примеров анализируются существование и устойчивость при изменении параметров некоторых основных типов распределений магнитного потока в трехслойном джозефсоновском контакте.

Работа выполнена в Лаборатории информационных технологий ОИЯИ.

Препринт Объединенного института ядерных исследований. Дубна, 2008

Christov I. G., Dimova S. N., Boyadjiev T. L.

E11-2008-160

Stability and Bifurcation of the Magnetic Flux Bound States

in Stacked Josephson Junctions

An effective numerical algorithm for solving the nonlinear system of ODE describing the static distributions of the magnetic flux in $N$-stacked Josephson Junctions (JJs), as well as the corresponding matrix Sturm-Liouville problem for studying their global stability, is proposed. The developed technique gives a possibility for detailed investigation of these multiparametric problems. We illustrate its application to analyze the existence, stability, lack of stability and some physical characteristics of two kinds of magnetic flux distributions in three-layered JJs.

The investigation has been performed at the Laboratory of Information Technologies, JINR. 


\section{STATEMENT OF THE PROBLEM}

In the last decades the propagation of electromagnetic waves in long Josephson Junctions (JJs) has been extensively studied both theoretically and experimentally (see, for example, [1-15]). Stacking the junctions may increase the usability of these devices especially for storage and transmission of information. Such structures make it possible to state and study new physical effects $[8,13]$ that do not occur in single $\mathrm{JJ}$ s.

In this paper we propose numerical technique for investigatig the properties of the solutions in the model of stacked JJs, based on inductively coupled layers [7]. The general case which takes into account both inductive and capacitive coupling is considered in [15].

A simple scheme of $N$-layered $\mathrm{JJ}$ is shown in Fig. 1, where black layers are barriers (insulators) and white layers are superconductors. In the symmetric case the electromagnetic interaction between junctions is represented only by a coupling constant $s[7,15]$.

The simplest generalizable model of stacked $\mathrm{JJ}$ is three-layered Josephson junction because it takes into account the difference in the behavior of the interior and exterior junctions. The first and the third junctions are coupled only to one neighboring junction, while the second junction is coupled to its two neighbors below and above. The numerical results, presented in this paper, are obtained mainly for the particular case of three-layered JJs, but the method of investigation and its program realization are developed for the general $N$-layered case $(N \geqslant 1)$.

The existence of Josephson current generates a specific magnetic flux [16]. When the external current is less than some critical value $\gamma_{\mathrm{cr}}$, the junction layers are in superconductive regime [16]. The transitions from superconductive to resistive regime are mathematically interpreted as bifurcation of the static distributions of the magnetic flux under the change of the parameters [17].

The vector of static distributions of the magnetic flux $\varphi(x)=\left(\varphi_{1}(x)\right.$, $\left.\varphi_{2}(x), \ldots, \varphi_{N}(x)\right)^{T}$ satisfies $[7,15]$ the nonlinear boundary value problem

$$
\begin{gathered}
-\varphi_{x x}+L\left(J_{z}+\Gamma\right)=0, \\
\varphi_{x}( \pm l)=H,
\end{gathered}
$$




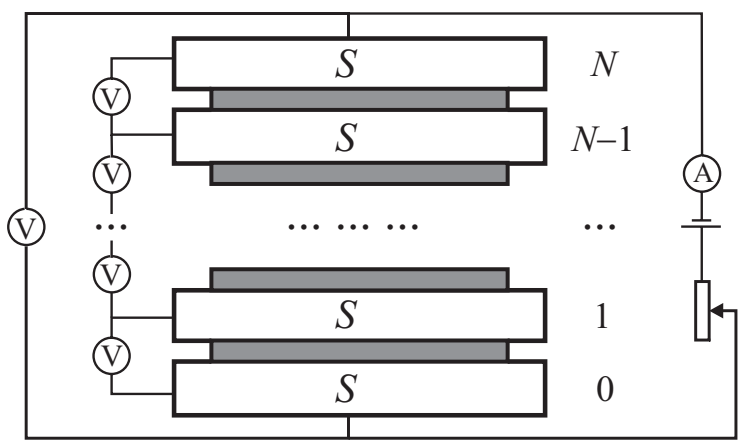

Fig. 1. $N$-layered JJ

where $J_{z}=\left(\sin \varphi_{1}, \sin \varphi_{2}, \ldots, \sin \varphi_{N}\right)^{T}$ is the vector of the Josephson current density, $\Gamma=\gamma(1,1, \ldots, 1)^{T}$ is the vector of the external current density, $H=$ $h_{e}(1,1, \ldots, 1)^{T}$ is the vector of the external magnetic field and $2 l$ is the length of the layers (the subscript $x$ means differentiation, the superscript $T$ means transposition). The matrix $L$ is a matrix of the inductive interaction $(-0.5<s \leqslant$ 0 for arbitrary $N)$ :

$$
L_{(N \times N)}=\left(\begin{array}{cccccc}
1 & s & 0 & \ldots & 0 & 0 \\
s & 1 & s & \ldots & 0 & 0 \\
. & . & . & \ldots & . & . \\
0 & 0 & 0 & \ldots & 1 & s \\
0 & 0 & 0 & \ldots & s & 1
\end{array}\right)
$$

\section{NUMERICAL METHOD}

In order to solve the nonlinear boundary value problem (1), we use an iterative algorithm, based on the continuous analog of Newton's method (CAMN) [19]. As initial approximations for the iteration process we take combinations (for the different layers) of solutions which exist in the one-layered case and $h_{e}=0$, $\gamma=0$ :

- Meissner solutions (denoted further by $M$ ) of the form $\varphi(x)=k \pi, k=$ $0, \pm 1, \pm 2, \ldots$;

- fluxon (antifluxon) solutions, for which there are exact analytical expressions in the case of infinite junctions $(l \rightarrow \infty)$ [23]. The single fluxon/antifluxon solution has the well-known form $\varphi(x)=4 \arctan \exp \{ \pm x\}+2 k \pi, k=$ $0, \pm 1, \ldots$ Further for $n$-fluxon distributions we use the simple notation $\Phi^{n}$, 
$n= \pm 1, \pm 2, \ldots$ For junctions of finite length, objects of type $\Phi^{n}$ are not fluxons in a strong sense, but by analogy the same terminology is used.

CANM gives a linearized boundary value problem at each iteration step:

$$
\begin{gathered}
-v_{x x}+L J_{z}^{\prime} v=\varphi_{x x}-L\left(J_{z}+\Gamma\right), \\
v_{x}( \pm l)=H-\varphi_{x}( \pm l)
\end{gathered}
$$

where $\varphi=\left(\varphi_{1}, \varphi_{2}, \ldots, \varphi_{N}\right)^{T}$ is the approximate solution found at the previous iteration step, $v=\left(v_{1}, v_{2}, \ldots, v_{N}\right)^{T}$ is the vector of the iteration corrections and $J_{z}^{\prime}(\varphi)$ is the matrix $\operatorname{diag}\left(\cos \varphi_{1}, \cos \varphi_{2}, \ldots, \cos \varphi_{N}\right)$.

The linear boundary value problems (2) are solved by means of Galerkin finite element method [18] and quadratic elements. The matrices of the corresponding linear algebraic problems are nonsymmetric. They are stored and used in sky-line form [20]. The linear algebraic problems are solved using $L U$-decomposition.

In order to study the global stability of the possible distributions of the magnetic flux, a matrix Sturm-Liouville problem [24] is generated:

$$
\begin{gathered}
-A u_{x x}+Q(x) u=\lambda u, \\
u_{x}( \pm l)=0, \\
\int_{-l}^{l}\langle u, u\rangle d x-1=0 .
\end{gathered}
$$

Here the matrix $A=L^{-1}$, and the potential $Q(x) \equiv J_{z}^{\prime}(\varphi(x))$. The minimal eigenvalue $\lambda_{\min }$ of (3) determines the stability of the distribution under consideration.

A minimal eigenvalue $\lambda_{\min }$ equal to zero means a bifurcation of this distribution caused by change of some parameter(s). For example, in infinite junction Meissner solution $M$ is stable, while $\Phi^{1}$ is quasistable (bifurcation solution with $\left.\lambda_{\min }=0\right)[21,22]$.

The finite element method is used to reduce the above matrix Sturm-Liouville problem (3) to a matrix algebraic problem, whose few smallest eigenvalues and the corresponding eigenfunctions are found by the subspace iteration method [20].

To test the accuracy of all realized methods, we have used the Runge method by computing the solutions on three embedded meshes. The numerous experiments made show a super-convergence of order four. As an example, in table the computed orders of convergence $\sigma$ of the smallest eigenvalue, as well as of the eigenfunction, corresponding to the first component of solution type $\left(\Phi^{1}, M, \Phi^{1}\right)$, $s=-0.3,2 l=10$, are shown. 
Order of convergence $\sigma(x)$ of EF, corresponding to solution type $\left(\Phi^{1}, M, \Phi^{1}\right)$, 1st component

\begin{tabular}{|c|c|c|c|c|}
\hline$x_{i}$ & $h=0.125$ & $h=0.0625$ & $h=0.03125$ & $\sigma$ \\
\hline-5 & 0.019669254 & 0.019669211 & 0.019669208 & 3.99 \\
\hline-4 & 0.027395953 & 0.027395900 & 0.027395896 & 3.99 \\
\hline-3 & 0.057938303 & 0.057938217 & 0.057938216 & 3.99 \\
\hline-2 & 0.139892955 & 0.139892807 & 0.139892798 & 3.99 \\
\hline-1 & 0.323331077 & 0.323330864 & 0.323330851 & 4.00 \\
\hline 0 & 0.486766104 & 0.486766424 & 0.486766444 & 4.02 \\
\hline 1 & 0.323331077 & 0.323330864 & 0.323330851 & 3.98 \\
\hline 2 & 0.139892955 & 0.139892807 & 0.139892798 & 3.98 \\
\hline 3 & 0.057938303 & 0.057938217 & 0.057938212 & 3.99 \\
\hline 4 & 0.027395953 & 0.027395900 & 0.027395896 & 3.99 \\
\hline 5 & 0.019669254 & 0.019669211 & 0.019669208 & 3.99 \\
\hline$\lambda$ & -0.002815748 & -0.002816608 & -0.002816662 & 4.00 \\
\hline
\end{tabular}

\section{NUMERICAL EXPERIMENT}

We briefly discuss some numerical results obtained by the developed algorithms. Especially we investigate numerically the static distributions of the magnetic flux and seek for critical values of the parameters $h_{e}$ and $\gamma$ where these distributions fail to exist.

3.1. Three-Layered Junctions. Because of a big variety of different triplets of solutions, in the present paper we explain results for $(M, M, M)$ and $\left(\Phi^{1}, M, \Phi^{-1}\right)$ solutions only.

In Figs. 2 and 3 the distributions of the internal magnetic field $\varphi_{x}(x)$ in the first layer of a three-layered junction are graphically shown. The results are for three different values of the external magnetic field $h_{e}(0$ and the two bifurcation values) when $\gamma=0, l=5$ and $s=-0.3$. Changing the value of $\gamma$ for given $h_{e}$ when the geometrical parameters $l$ and $s$ are fixed, we get the region of existence of the corresponding solution in the plane $P \equiv\left(h_{e}, \gamma\right)$. We expected a symmetry of this region with respect to $h_{e}$ and $\gamma$ for solutions of types $(M, M, M)$ and $\left(\Phi^{1}, M, \Phi^{-1}\right)$, and this was confirmed by the numerical results. In Figs. 4 and 5 the regions of existence $\left(h_{e} \geqslant 0\right)$ in the plane $P$ for the same solutions are shown. Every point on these curves is a bifurcation point and, consequently, satisfies

$$
\lambda_{\min }\left(\gamma_{\mathrm{cr}}, h_{e}\right)=0
$$

The main physical characteristics of interest - energy, number of fluxons, full magnetic flux - are computed as well. The dependence of these characteristics on the geometric parameters of the junction is investigated and new interesting 


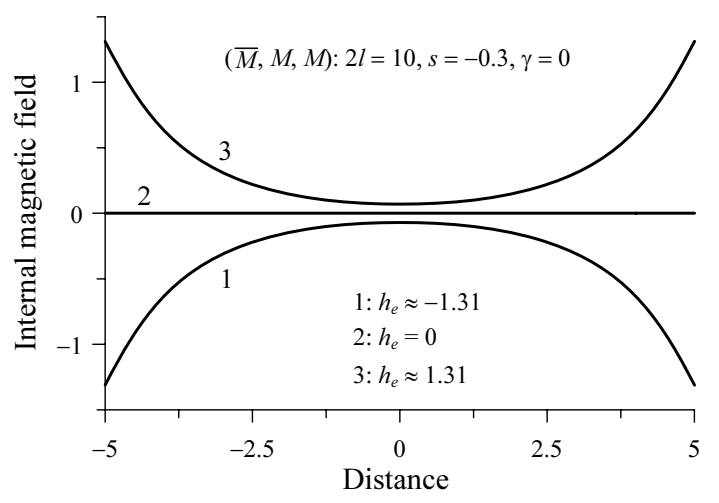

Fig. 2. Solution of type $(M, M, M)$

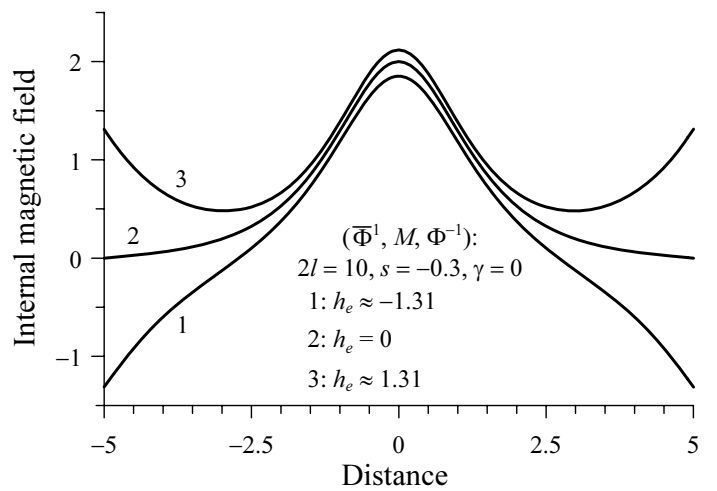

Fig. 3. Solution of type $\left(\Phi^{1}, M, \Phi^{-1}\right)$

results are found. Let us denote by $\Delta \varphi$ the vector of full magnetic flux through the junction:

$$
\Delta \varphi=\left(\Delta \varphi_{1}, \ldots, \Delta \varphi_{N}\right)^{T}, \quad \Delta \varphi_{i}=\varphi_{i}(l)-\varphi_{i}(-l) .
$$

Equations (1) can be considered as necessary conditions for extremum of the functional of full energy $F[\varphi]$ of the junction:

$$
F[\varphi]=\int_{-l}^{l}\left[\frac{1}{2}\left\langle\varphi_{x}, A \varphi_{x}\right\rangle+U(\varphi)\right] d x-\langle A H, \Delta \varphi\rangle
$$




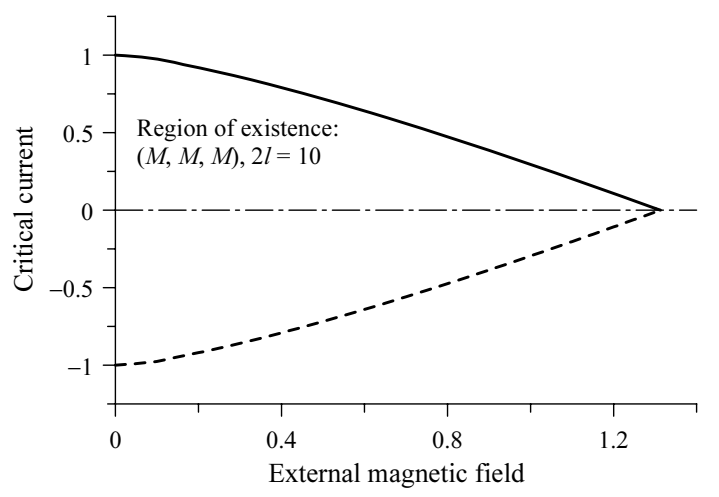

Fig. 4. Region of existence for $(M, M, M)$

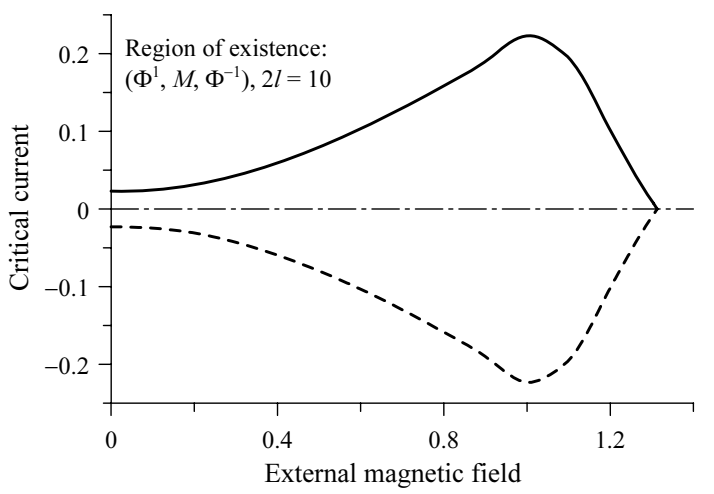

Fig. 5. Region of existence for $\left(\Phi^{1}, M, \Phi^{-1}\right)$

where the density $U=\sum_{i=1}^{N} U_{i}\left(\varphi_{i}\right), U_{i}=1-\cos \varphi_{i}+\gamma \varphi_{i}$. Equation (1a) is the Euler-Lagrange equation for (4) and the boundary conditions (1b) follow from Weierstrass-Erdmann conditions.

The full energy $F$ is presented as a sum of the energies $F_{i}, i=1, \ldots, N$ of the non-interacting layers and the coupling energy $F_{\text {int }}$ :

$$
F=\sum_{i=1}^{N} F_{i}+F_{\mathrm{int}} .
$$

For this reason the matrix $A$ is presented as a sum $A=I+B$, where $I$ is the 
identity matrix. For the full energy this gives

$$
\begin{aligned}
F[\varphi]=\int_{-l}^{l}\left[\frac{1}{2}\left\langle\varphi_{x}, \varphi_{x}\right\rangle+\frac{1}{2}\left\langle\varphi_{x}, B \varphi_{x}\right\rangle+U(\varphi)\right] & d x- \\
& -\langle I H, \Delta \varphi\rangle-\langle B H, \Delta \varphi\rangle .
\end{aligned}
$$

Then

$$
\begin{gathered}
F_{i}\left[\varphi_{i}\right]=\int_{-l}^{l}\left[\frac{1}{2} \varphi_{i, x}^{2}+U_{i}\left(\varphi_{i}\right)\right] d x-h_{e} \Delta \varphi_{i}, \\
F_{\mathrm{int}}[\varphi]=\frac{1}{2} \int_{-l}^{l}\left\langle\varphi_{x}, B \varphi_{x}\right\rangle d x-\langle B H, \Delta \varphi\rangle .
\end{gathered}
$$

A comparison of the dependence $F(\gamma)$ for solutions of type $(M, M, M)$ (see curve 1 ) and $\left(\Phi^{1}, M, \Phi^{-1}\right)$ (curve 2 ) is made in Fig. 6. The values of $F(\gamma)$ are computed by formulae (5). The boundary points $B_{1}$ and $B_{2}$ are bifurcation points for the parameter $\gamma$. The curve 1 lies under curve 2, which means that for given $h_{e}=1$ the distribution $(M, M, M)$ has bigger critical current (by modulus) than the distribution $\left(\Phi^{1}, M, \Phi^{-1}\right)$.

Let us mention the different behavior of the dependence $F_{123}(\gamma)$ for the two distributions (see Fig. 7). When $|\gamma|$ increases, the coupling energy $F_{123}(\gamma)$

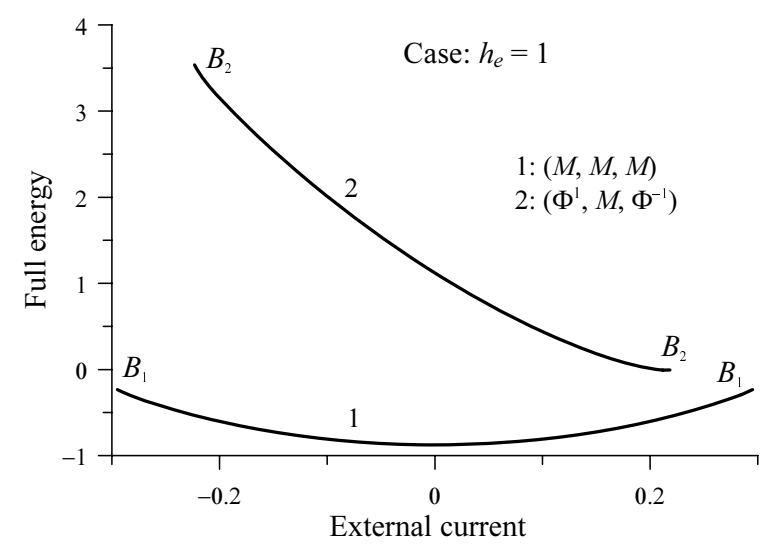

Fig. 6. Full energy $F(\gamma)$ for $(M, M, M)$ - and $\left(\Phi^{1}, M, \Phi^{-1}\right)$-type solutions 
for $(M, M, M)$-distribution monotonically decreases in contrast to $F_{123}(\gamma)$ for $\left(\Phi^{1}, M, \Phi^{-1}\right)$ which monotonically increases.

Finally, in Fig. 8 a comparison of the energy $F_{2}(\gamma)$ of the middle layer for $(M, M, M)$ and $\left(\Phi^{1}, M, \Phi^{-1}\right)$ solutions is shown. It is clearly seen that the changes $M \rightarrow \Phi^{1}$ and $M \rightarrow \Phi^{-1}$ in the external layers result mainly in decreasing of the region of existence with respect to external current $\gamma$.

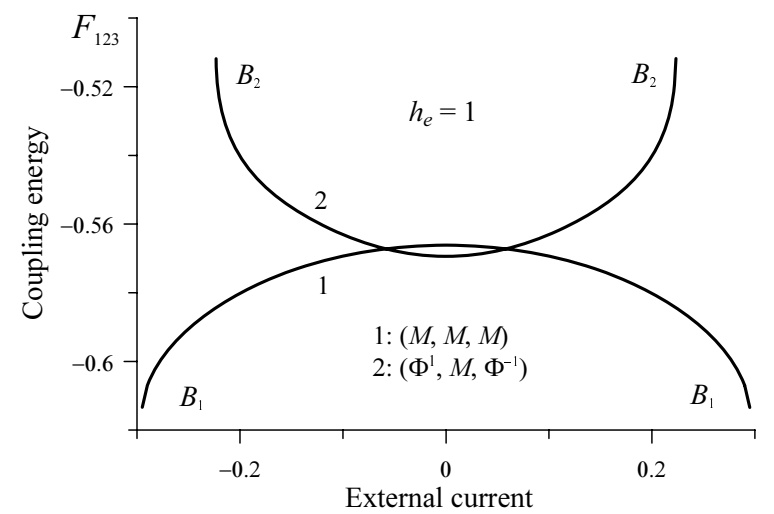

Fig. 7. Coupling energy $F_{123}(\gamma)$ for $(M, M, M)$ - and $\left(\Phi^{1}, M, \Phi^{-1}\right)$-type solutions

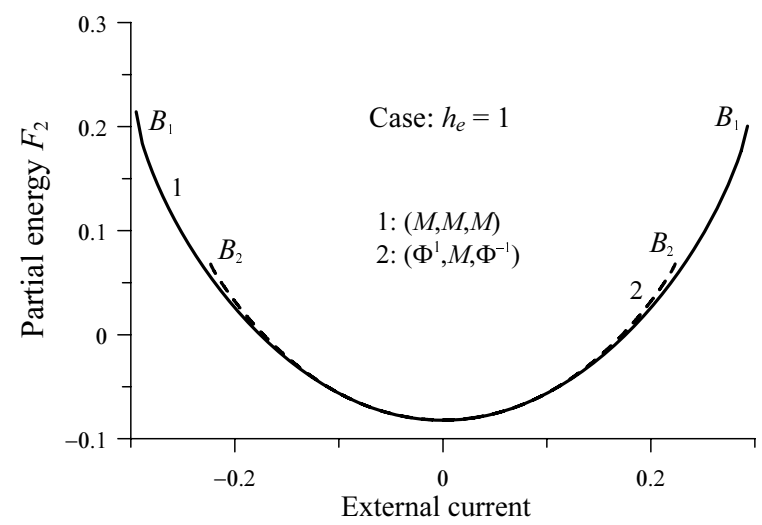

Fig. 8. Partial energies $F_{2}(\gamma)$ for $(M, M, M)$ - and $\left(\Phi^{1}, M, \Phi^{-1}\right)$-type solutions 
3.2. Single Josephson Junction. In the process of investigation some interesting observations were made about the behavior of the solutions depending on the length of the junction. For their deeper understanding we analyzed the case of single junction, which corresponds to $s=0$.

The numerical experiments have shown that when the length $2 l$ grows, the supports of the bifurcation curves for fluxons $\Phi^{1}, \Phi^{2}, \Phi^{3}, \ldots$ tend consequently to the support of the bifurcation curve for Meissner solution. This fact is illustrated in Figs. 9 and 10. One can see that the support of the bifurcation curve for $\Phi^{1}$ (Fig. 9, $2 l=14$ ) and for $\Phi^{1}, \Phi^{2}, \Phi^{3}$ (Fig. 10, $2 l=30$ ) coincides within the plotting resolution with the support of the bifurcation curve for Meissner solution. And what is more, the maximal critical current in these cases lies on the same curve.

The numerical observations gave a hint to ask for analytical dependencies. The following ones are found:

1. Let $\left(\alpha\left(\Phi^{k}, l\right), \beta\left(\Phi^{k}, l\right)\right)$ be the interval in $h_{e}$ for $\gamma=0$ and fixed halflength $l$, for which the solution $\Phi^{k}$ is stable. Then

$$
\alpha\left(\Phi^{k}, l\right) \searrow 0 \quad \text { when } \quad l \rightarrow \infty, \quad \beta\left(\Phi^{k}, l\right) \searrow 2 \quad \text { when } \quad l \rightarrow \infty .
$$

2. Let $(-\alpha(M, l), \alpha(M, l))$ be the interval in $h_{e}$ for $\gamma=0$ and fixed halflength $l$, for which the solution $M$ is stable. Then

$$
\alpha(M, l) \searrow 2 \text { when } \quad l \rightarrow \infty
$$

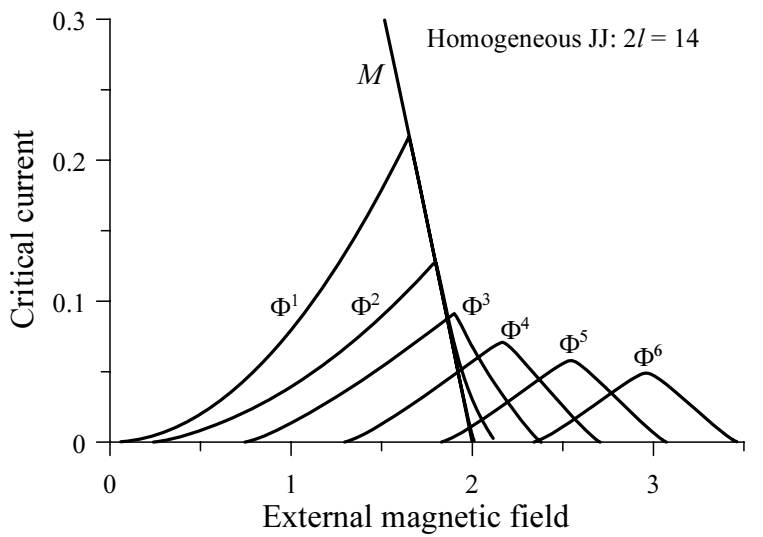

Fig. 9. Bifurcation curves for $2 l=14$ 


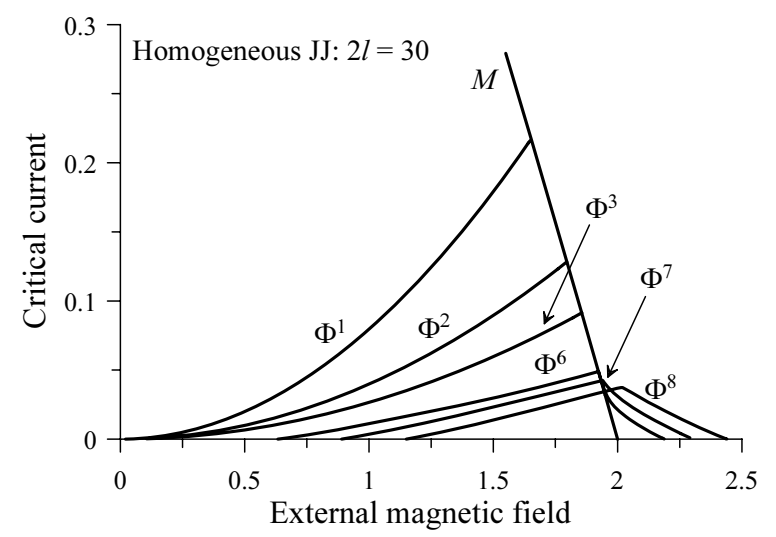

Fig. 10. Bifurcation curves for $2 l=30$

3. For arbitrary $l$ the following inequalities hold:

$$
\begin{gathered}
\alpha\left(\Phi^{1}, l\right)<\alpha\left(\Phi^{2}, l\right)<\alpha\left(\Phi^{3}, l\right)<\ldots, \\
\alpha(M, l / 2)=\beta\left(\Phi^{1}, l\right)<\beta\left(\Phi^{2}, l\right)<\beta\left(\Phi^{3}, l\right)<\ldots, \\
\alpha\left(\Phi^{m}, l\right)=\alpha\left(\Phi^{k}, \frac{k}{m} l\right), \quad \beta\left(\Phi^{m}, l\right)=\beta\left(\Phi^{k}, \frac{k+1}{m+1} l\right), k \geqslant m .
\end{gathered}
$$

4. If for $h_{e} \leqslant 2, \gamma=0$ and fixed $l$ the solution $\Phi^{k}$ is stable, then $\Phi^{1}, \Phi^{2}, \ldots$, $\Phi^{k-1}$ are stable as well.

Let us denote by

$$
N[\varphi]=\frac{1}{2 l \pi} \int_{-l}^{l} \varphi(x) d x
$$

the average value of the bound state $\varphi(x)$ on the junction [19]. Functional (6) depends on all of the parameters of the model. In Fig. 11 the dependence $N\left(h_{e}\right)$ for the bifurcation solutions $\Phi^{-1}, M, \Phi^{1}, \Phi^{2}$ and $\Phi^{3}$ is presented in the case of single JJ of full length $2 l=10$. The solid lines correspond to $\gamma_{\mathrm{cr}} \geqslant 0$, the dashed ones - to $\gamma_{\mathrm{cr}} \leqslant 0$. Note that for $\gamma_{\mathrm{cr}}=0$ the values of $N[\varphi]$ are integer numbers: $N\left[\Phi^{n}\right]=n, N[M]=0$. When $\gamma_{\mathrm{cr}} \neq 0$ the following relations are fulfilled:

$$
N\left[\Phi_{+}^{n}\right]+N\left[\Phi_{-}^{n}\right]=2 n, \quad N\left[M_{+}\right]+N\left[M_{-}\right]=0,
$$

where the subscripts \pm correspond to the sign of $\gamma_{\mathrm{cr}}$. 


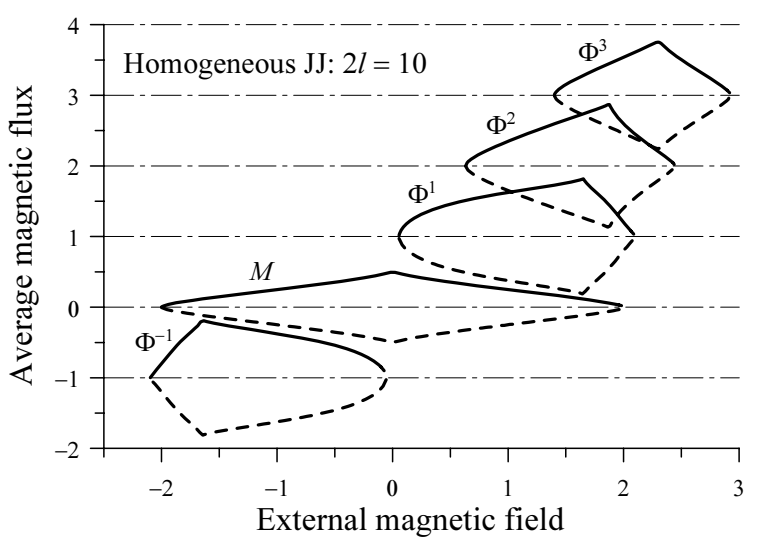

Fig. 11. The number of fluxons in single JJ

The above-mentioned facts can be considered as conservation laws of the average magnetic flux. So formula (6) determines the admissible number of fluxons in the solutions of problem (1), having fixed sign of $\varphi_{x}(x)$.

\section{CONCLUSIONS}

Effective numerical algorithms are worked out for solving the nonlinear system of ODE for finding the static distributions of the magnetic flux in $N$-stacked JJs, as well as the corresponding matrix Sturm-Liouville problem for studying their global stability. The developed technique gives a possibility for detailed investigation of these multiparametric problems. We illustrate its application to analyze the existence, stability, lack of stability and some physical characteristics of two types of magnetic flux distributions in three-layered JJs. In addition, some new numerical and analytical results for one-layered JJs are found.

Acknowledgements. This work is supported by Sofia University Scientific Foundation under Grant No. 135/2008.

\section{REFERENCES}

1. M. B. Mineev, G. S. Mkrtchjan, V. V. Schmidt, J. Low Temp. Phys. 45 (1981) 497.

2. Yu. S. Kivshar, B. A. Malomed, Phys. Rev. B 37 (1988) 9325.

3. A. F. Volkov, Solitons in Josephson superlattices, JETP Lett. 45 (6) (1987) 299-301. 
4. N. Gronbech-Jensen, M. R. Samuelsen, P.S. Lomdahl, J. A. Blackburn, Phys. Rev. B 42 (1990) 3976.

5. A.V. Ustinov, Solitons in Josephson junctions, Physica D 123 (1998) 315-329.

6. T.L. Boyadjiev, D. V.Pavlov, I. V.Puzynin, Computation of Bifurcations of Stable States in Two-Layer Inhomogeneous Josephson Junctions, JINR Comm. P5-89-173, Dubna, 1989.

7. S. Sakai, P. Bodin, N.F. Pedersen, Fluxons in thin-film superconductor-insulator superlattices, J. Appl. Phys. 73 (5) (1993) 2411-2418.

8. I. P. Nevirkovets, J.E. Evetts, M. G. Blamire, Transition from Single Junction to Double Junction Behavior in Sisis-Type Nb-Based Devices, Phys. Lett. A 187 (1) (1994) 119.

9. L. N. Bulaevskii, M.Zamora, D. Baeriswyl, H. Beck, John R. Clem, Time-dependent equations for phase differences and a collective mode in Josephson-coupled layered superconductors, Phys. Rev. B 50 (1994) 12831-12834.

10. R. Kleiner, P. Muller, H. Kohlstedt, N. F. Pedersen, S. Sakai, Phys. Rev. B 50 (1994) 3942-3952.

11. S. N. Song, P. R. Auvil, M. Ulmer, and J. B. Ketterson, Vortex structure and Josephson supercurrent in stacked double Josephson junctions, Phys. Rev. B 53 (10) (1996) R6018.

12. V.M. Krasnov and D. Winkler, Static and dynamic properties of stacked Josephson junctions: Analytic solution, Phys. Rev. B 56 (1997) 9106-9115.

13. E. Goldobin, A. V. Ustinov, Current locking in magnetically coupled long Josephson junctions, Phys. Rev. B 59 (17) (1999) 11532-11538.

14. P. Kh. Atanasova, T. L. Bojadjiev, and S. N. Dimova, Numerical Simulation of Critical Dependences for Symmetric Two-Layered Josephson Junctions, Comp. Math. Math. Phys. 46 (4) (2006) 666-679.

15. M. Machida, S. Sakai, Unifed theory for magnetic and electric field coupling in multistacked Josephson junctions, PRB 70 (2004) 144520.

16. K. K. Licharev, Dynamics of Josephson Junctions and Circuits, Gordon and Breach, New York, 1986.

17. Yu. S. Gal'pern, A. T. Filippov, Bound states of solitons in inhomogeneous Josephson junctions, Sov. Phys. JETP 59 (1984).

18. V. Thomee, Galerkin finite element method for parabolic problems. Berlin: Springer, 1997. 
19. I. V.Puzynin et al., Methods of computational physics for investigation of models of complex physical systems, Particles \& Nuclei 38 (1) (2007).

20. K. J.Bathe and E. Wilson, Numerical Methods in Finite Element Analysis, Prentice Hall, Englewood Cliffs, 1976.

21. J. Rubinstein, Sine-Gordon equation, J. Math. Phys. 16 (1970) 96.

22. M. B. Fogel et al., Dynamics of sine-Gordon solitons in the presence of perturbations, Phys. Rev. B 15 (1977) 3.

23. I. D. Iliev, E. Kh. Khristov, and K.P. Kirchev, Spectral methods in soliton equations, Longman Sci. \& Techn.; Wiley, 1994.

24. M.A. Naimark, Linear differential operators, Nauka, Moscow, 1969.

Received on November 17, 2008. 


\section{Редактор Е. И. Кравченко}

Подписано в печать 24.12.2008.

Формат $60 \times 90 / 16$. Бумага офсетная. Печать офсетная.

Усл. печ. л. 0,81. Уч.-изд. л. 1,01. Тираж 310 экз. Заказ № 56457.

Издательский отдел Объединенного института ядерных исследований 141980 , г. Дубна, Московская обл., ул. Жолио-Кюри, 6.

E-mail: publish@jinr.ru www.jinr.ru/publish/ 